Review articles

Med Klin Intensivmed Notfmed 2020 • 115 (Suppl 1):S4-S9

https://doi.org/10.1007/s00063-020-00673-4

Received: 12 October 2019

Accepted: 14 January 2020

Published online: 31 March 2020

(c) The Author(s) 2020

\section{Redaktion}

M. Bauer, Jena

M. Singer, London

\section{Introduction}

Infections reflect frequent and typically self-limiting events. A proportion of infected patients, however, develop organ dysfunction through a dysregulated host response to infection, i.e., sepsis [40]. Research aimed to elaborate the defense mechanisms against infections, clearly focused on lowering the pathogen burden through resistance, i.e., the ability of the organism to detect and to destroy the invading microorganisms. During the past years an alternative defense mechanisms came into focus: Disease tolerance, mainly characterized by regenerative responses, which allow retaining cell and organ functions of the infected host without lowering the pathogen burden.

\section{Failing resistance and tolerance in sepsis}

An overwhelming resistance response of the immune system in sepsis has been hypothesized for a century and found a convincing molecular explanation in 1985 by Bruce Beutler and colleagues [7]: They observed tissue damage in sepsis and firstly interpreted this phenomenon as an overreaction of immune cells using their antibacterial effector molecules leading to "collateral damage". Understanding of sepsis as a systemic (hyper-)inflammatory response (SIRS) to pathogenic infections prevailed the interpretation of sepsis-associated organ dysfunction for the last 3 decades.

During the past years reinterpretation of sepsis pathogenesis was provoked by

\author{
M. Bauer ${ }^{1,2} \cdot$ R. Wetzker \\ ' Department of Anesthesiology and Intensive Care Medicine, Jena University Hospital, Jena, Germany \\ ${ }^{2}$ Integrated Research and Treatment Center Center for Sepsis Control and Care (CSCC), Jena University \\ Hospital, Jena, Germany
}

\title{
The cellular basis of organ failure in sepsis-signaling during damage and repair processes
}

a phenomenon occurring in later stages of the disease. The majority of patients who survive the initial phase of sepsis suffer from impaired innate and acquired immunity up to the occurrence of secondary infections or reactivation of dormant viruses [8].

There is increasing evidence that this phenomenon might be interpreted as uncontrolled tolerance. The normally balanced relation of damaging effects of microbial pathogens in host tissue and the associated maintenance responses seemingly go out of control. The repair capacity of the affected organs becomes increasingly exhausted.

Hence both failing resistance and tolerance responses contribute to the phenotypes of sepsis ( $\bullet$ Fig. 1 ). Notably, in septic patients evidence for all aspects of immune dysfunction, i.e., predominant hyperinflammatory responses ("SIRS"), patients with predominant immunoparalytic characteristics ("CARS") and also mixed patterns ("MARS") have been reported $[8,19,33]$.

The seminal role of resistance and tolerance in the adaptive responses of higher organisms to infectious attacks raises the question for the origin and causes of these differential response patterns, in particular when the host is repeatedly confronted with pathogens and their pathogen-associated molecular patterns (PAMPs).

Many data indicate differential ability of specific pathogens or PAMPs to induce alternative immune responses. Thus ample studies reveal lipopolysaccharides (LPS) as constituents of gram negative bacteria as typical inducers of decreased release of proinflammatory cytokines from affected immune cells unveiling LPS as typical mediators of (endotoxin) tolerance [45]. Vice versa $\beta$-glucan as a component of fungal cell membranes has been frequently shown to induce enduring increase of the release of proinflammatory cytokines from immune cells, characterizing $\beta$-glucan as prototypical inducer of resistance responses [32].

This simple scheme is considerably challenged by recent observations of consecutive appearance of resistance and tolerance responses in dependence on the concentration of PAMPs. For instance, Maitra et al. revealed increased resistance responses of monocytes after treating monocytes with very low doses of LPS [27]. Similar stimulation of proinflammatory cytokine release at extremely low doses of LPS-i.e. increased resistance-and inhibition at high LPS doses-i.e. tolerance-has been shown in a study of microglia response pattern to PAMPs [21]. Consequently, both pathogen species and pathogen dose might determine resistance or tolerance responses of the host [6].

The complex differential response patterns of the immune system and other organs during sepsis necessitate careful clinical monitoring, i.e. personalization of care. While already a reality in the field of oncology, the time critical decisions in intensive care medicine have so far hindered to take functional characteristics of resistance and tolerance responses of the immune system into account. Thus, the need for better diagnostic tests has been 


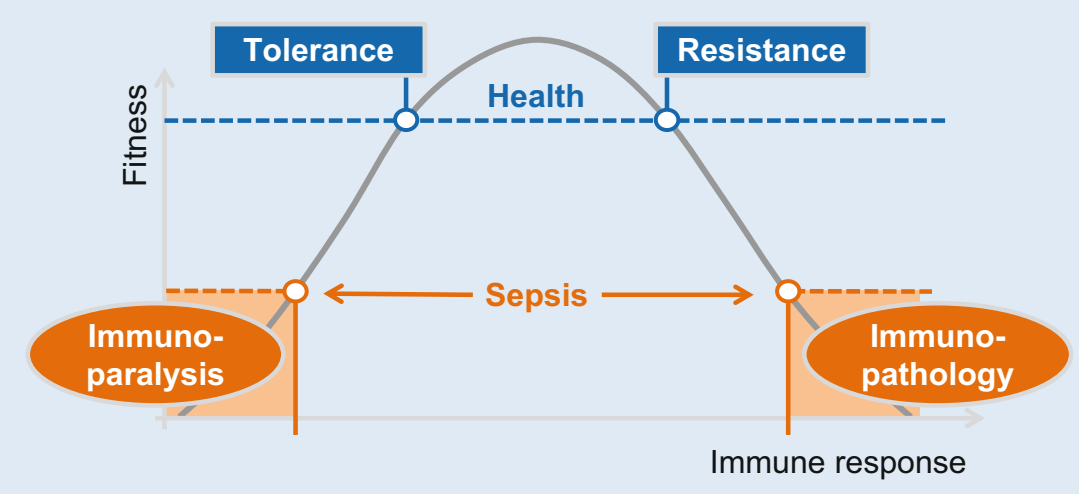

Fig. $1 \Delta$ Fitness of the host and immune response to infections

voiced for infectious diseases in general and with special emphasis on sepsis [15] and seems feasible with modern diagnostic platforms. We and others have shown that compound biomarker panels that consist of several transcripts reflecting both the proinflammatory and antiinflammatory response can be used at the point-of-care to provide additional information on the host response compared to conventional biomarkers $[4,30]$ allowing to discriminate various phenotypes of sepsis. In addition, recent evidence would suggest that pattern recognition with artificial intelligence on routine parameters might help to discriminate inflammatory or metabolic phenotypes with potential impact on treatment [38].

\section{Metabolic reflections of resistance and tolerance responses}

The biological phenomena of resistance and tolerance responses of the immune system are closely linked to energy resources and metabolic processes in the cells involved. In general resistance responses reveal anabolic, i.e. energy-consuming metabolic traits, whereas tolerance predominantly implicates catabolic, i.e. energy providing processes [6].

The energy-consuming character of anabolic resistance responses can be illustrated by the resource needs of the clonal expansion of adaptive immune cells secondary to infection. Doing so, the immune system and other organs involved in the resistance response strongly com- pete for energy resources with other anabolic functions.

In contrast to the energy wasting resistance responses, immune tolerance and in a broader sense organismic tolerance represents an energy saving response pattern of the organism to infection [2]. During tolerance responses the titer of invading microorganism does not change significantly and cells and organs involved tend to react passively by building barriers for microorganism dissemination and/or regenerating cells and their organelles, which are damaged by the pathogens [2].

Recent studies provide evidence that overwhelming immune tolerance leads to immunoparalysis (• Fig. 1). Experimentally, Grondman et al. showed tight association of endotoxin-induced immune tolerance with loss of monocyte metabolic plasticity and reduction of oxidative burst with leading to microbial dissemination [18].

It becomes apparent in all these studies that metabolic processes are closely interlinked to damage and repair processes in cells and tissues [5]. The homeostasis of synthesis and formation of cellular organelles on one site and their degradation is an essential prerequisite for vitality of the cells and, thus, integrity of tissue and organs.

\section{Signaling and targeting of resistance and tolerance}

Efficient adaptation to environmental challenges is key for survival and conserved throughout evolution. Conse- quently anabolic resistance- and catabolic tolerance responses need tight linkage to the energy metabolism of cells involved. To manage this goal all cells possess sensors for the energy state, most notably mechanistic target of rapamycin (mTOR) and AMP-activated protein kinase (AMPK) [6]. Both ubiquitous signaling proteins have been evolved during evolution over the last 2 billion years as key mediators of metabolic responses [37].

Notably, mTOR acts as a go-between the growth promoting effects of insulin. In doing so mTOR works in tight cooperation with its upstream mediators PI3K and Akt [35]. Rapamycin, a drug which directly interacts with mTOR, blocks its signaling effects and was shown to exhibit antiproliferative effects specifically on immune cells leading to its approval as immunosuppressive drug [42]. Consistent with the aforementioned concept, recent clinical studies reveal a significant decrease in inflammation and senescence markers in patients after treatment with mTOR inhibitors [24].

Some of the regenerative effects of rapamycin might be explained by its strong ability to induce autophagy [26]. Autophagy has been defined as stress response pathway mediating the breakdown of malfunctioning cellular material and initiating cell content recycling [36]. The central mediator of cellular recycling activities is AMPK [43].

AMPK acts as highly conserved signaling sensor activated by elevated AMP/ADP concentration [31]. Consequently, AMPK is activated by caloric restriction and deactivated by nutritional overload opposing mTOR, which is stimulated at nutrient abundance and anabolic conditions [37]. AMPK can also be activated by a number of energyconsuming stress responses including physical activities and infection [13]. Thus low doses of ionizing radiation were shown to induce a radioprotective effect by promoting DNA repair and cell survival signaling proteins through activating AMPK and inhibiting mTOR signaling [46].

Several studies suggest AMPK as a possible drug target in aging. Thus, treatment with metformin, an AMPK 
Med Klin Intensivmed Notfmed 2020 - 115 (Suppl 1):S4-S9 https://doi.org/10.1007/s00063-020-00673-4

(c) The Author(s) 2020

\section{Bauer $\cdot$ R. Wetzker}

\section{The cellular basis of organ failure in sepsis—signaling during damage and repair processes}

\section{Abstract}

Sepsis is defined as life-threatening organ dysfunction caused by a dysregulated host response to infection. This definition, updated in 2016, shifted the conceptual focus from exclusive attention to the systemic inflammatory response toward the multifactorial tissue damage that occurs during the progression of infection to sepsis and shock. Whereas targeting the inflammatory host response to infection did not translate into improved clinical management of sepsis, recent findings might shed new light on the maladaptive host-pathogen interaction in sepsis and pave the way for "theranostic" interventions. In addition to the well-known resistance responses of the immune system that result in pathogen clearance, "disease tolerance" has recently been acknowledged as a coping mechanism of presumably equal importance. We propose that both defense mechanisms, "resistance" and "disease tolerance", can get out of control in sepsis. Whereas excessive activation of resistance pathways propagates tissue damage via immunopathology, an inappropriate "tolerance" might entail immunoparalysis accompanied by fulminant, recurrent or persisting infection. The review introduces key signaling processes involved in infection-induced "resistance" and "tolerance". We propose that elaboration of these signaling pathways allows novel insights into sepsis-associated tissue damage and repair processes. Moreover theranostic opportunities for the specific treatment of sepsis-related hyperinflammation or immunoparalysis will be introduced. Agents specifically affecting either hyperinflammation or immunoparalysis in the course of sepsis might add to the therapeutic toolbox of personalized care in the field of organ dysfunction caused by infection. (This article is freely available.)

Keywords

Sepsis · Resistance $\cdot$ Immunopathology Disease tolerance $\cdot$ Immunoparalysis

\section{Die zelluläre Basis des Organversagens bei Sepsis - Signalwege in Gewebeschädigung und Reparaturprozessen}

\section{Zusammenfassung}

Sepsis ist definiert als lebensbedrohliche Organfunktionsstörung infolge einer gestörten Wirtsantwort auf eine Infektion. Diese Definition, 2016 aktualisiert, verlagerte den konzeptionellen Fokus von der Entzündungsreaktion des Wirts zur multifaktoriellen Gewebeschädigung, die während des

Fortschreitens der Infektion zur Sepsis auftritt. Während die therapeutische Intervention in die Entzündungsreaktion des Wirts auf eine Infektion nicht zu einem verbesserten klinischen Management der Sepsis führte, werfen jüngste Erkenntnisse ein neues Licht auf die maladaptive Wechselwirkung zwischen Wirt und Krankheitserreger bei der Sepsis. Zusätzlich zu den bekannten Resistenzreaktionen des Immunsystems, die zur
Beseitigung von Krankheitserregern führen, wird zunehmend die "disease tolerance" oder „Resilienz" als ein Bewältigungsmechanismus von vermutlich ähnlicher Bedeutung erkannt. Wir diskutieren im vorliegenden Review, dass sowohl die "Resistenz" als auch die "Resilienz" bei Sepsis außer Kontrolle geraten können. Während eine übermäßige Aktivierung von Resistenzreaktionen zur Immunpathologie mit konsekutiver Gewebeschädigung führt, kann eine unangemessene Resilienz wahrscheinlich in eine Immunparalyse mit fulminanten, rekurrierenden oder persistierenden Infektionen münden. Wir diskutieren zudem wichtige Signalprozesse, die an der Vermittlung infektionsbedingter Resistenz- und Resilienzmechanismen beteiligt sind. Wir denken, dass diese Signalwege neue Möglichkeiten zur Therapie der sepsisassoziierten Gewebeschädigung und zur Stimulation von Reparaturprozessen eröffnen. Darüber hinaus werden neue Ansätze zur gezielten Behandlung von sepsisbedingter Hyperinflammation oder Immunparalyse vorgestellt, die das Portfolio für die personalisierte Versorgung der durch Sepsis verursachten Organfunktionsstörung erweitern sollten.

Schlüsselwörter

Sepsis · Resistenz $\cdot$ Immunpathologie . Toleranz $\cdot$ Immunparalyse activator, can extend lifespan in several species, e.g. Caenorhabditis elegans and mice $[14,28]$. Because of its lifeextending effects in several species, as well as its anti-inflammatory and antidiabetic activities, metformin, along with rapamycin, has been approved for the clinical trial targeting aging with metformin (TAME) in elderly (clinicaltrials.gov).

mTOR has been characterized as a potent inhibitor of autophagy [1]. Vice versa AMPK can inhibit the activity of mTOR complex (mTORC1) via two different mechanisms, either by directly phosphorylating Raptor, a regulatory component of mTORC1, or by the phosphorylation of tuberous sclerosis protein 2 (TSC2) [25]. High fat diet and obesity lead to increased inflammation, macrophage activation and downregulation of AMPK [17]. Increased inflammation is one of the main causes of tissue deterioration with age. As previously discussed, activation of AMPK can reduce oxidative stress, decrease endoplasmatic reticulum stress and inflammation [20]. Notably, AMPK activation suppresses the signal- ing of anabolic insulin/IGF-1 pathway underlining again the opposing functions of mTOR and AMPK in the control of anabolic and catabolic processes [35].

The opposing functions of mTOR and AMPK in the control of resistance and tolerance responses can be summarized in a signaling scheme, which also involves mediators "upstream" and "downstream" of these central sensors of cellular energy level (• Fig. 2).

The predominant role of (pathogen) stress dose on the development of resistance and tolerance responses of im- 


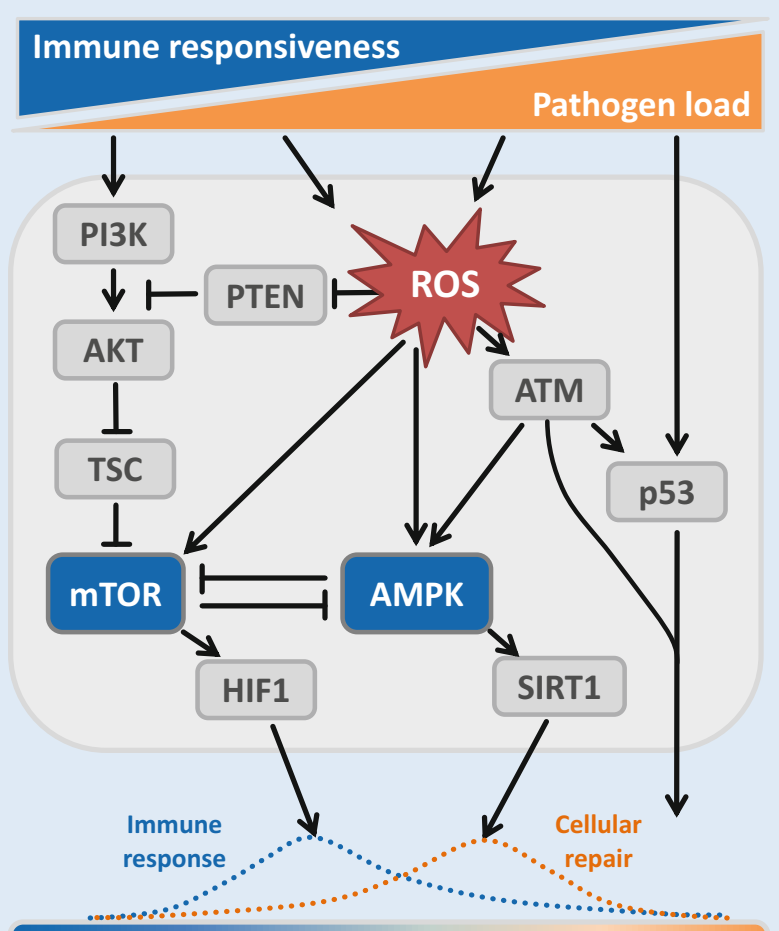

Resting - Resistance - Tolerance - Damage
Fig. $2<$ Signaling scheme reflecting signaling cascades of resistance and tolerance as a function of pathogen burden. Adapted from [6] mune cells has been outlined. Increasing pathogen stress is progressively exhausting energy resources of the immune system leading to decreasing "immune responsiveness" and concomitant tolerance responses. All stressors are inducing adaptive responses, which provoke energy deficit.

\section{How can these concepts be applied in sepsis and organ failure?}

Recent observations in rodent sepsis models revealed novel therapeutic approaches to modulate the opposing phenotypes of altered immunity in sepsis. Probably the most surprising impulses to treat sepsis came from experiments investigating cross effects of environmental stressors on the response patterns of septic mice.

In a seminal study Figureido et al. [16] screened a large number of chemicals, including anthracyclines, for their effects on inflammatory reactions of a macrophage cell line. These cytotoxins-commonly applied for the therapy of solid cancers-were injected into mouse models of polymicrobial sepsis. In comparison to the controls, a significantly lower mortality of the toxintreated animals was observed. Interestingly, whole body irradiation produced similar effects. More detailed investigations revealed stimulation of DNA repair mechanisms in response to trace amounts of anthracyclines with induction of autophagy. Together these data indicate that stressors like toxins or irradiation induce repair mechanisms, which suppress immunopathology thus preventing tissue damage. Interestingly, dietary restriction as another environmental stressor could suppress hyperinflammatory responses in septic mice [41]. Sepsis-induced mortality could be abolished by three weeks of moderate fasting prior to provoking sepsis by a polymicrobial cocktail or by LPS injection. In addition to conventional use of anti-inflammatory drugs different kinds of stressors might be applied to suppress inflammation-induced damage.

The anti-inflammatory effects of anthracylines, of radiation and food deprivation can be explained as a consequence of their energy-demanding effects. En- vironmental stressors and inflammatory reactions inside the immune cells compete for the same energy sources and consequently protein synthesis and cell proliferation. It can be supposed that treatment of cells with anthracylines and radiation or food deprivation provoke a turn of the immune responses towards tolerance reactions. Morbidity induced by systemic inflammation decreases and the organism exhibits "disease tolerance".

What concepts arise regarding immunoparalysis and damage induced by disseminating microbes? There are initial hints that proinflammatory cytokines can be used to induce arousal of a "dormant" immune system. Leentjens et al. demonstrated partial reversion of immunoparalysis of humans by treatment with IFN $\gamma$ in vivo [22]. The immunostimulatory agents used in these models cover a broad spectrum of agents from Candida albicans and its cell wall component $\beta$-glucan [32], BCG vaccine [11] and surprisingly also acetylsalicylic acid (ASA) [23].

An overview regarding opportunities to modulate immunity as it relates to cellular energy stores is shown in - Fig. 3. In addition to direct immune-modulating agents, environmental stressors like low doses of cytotoxic agents, radiation or deprivation of food seem to enter translational research and could potentially explain observations in clinical trials, e.g. regarding the impact of caloric restriction on outcome in the ICU [10].

Taken together, interventions into anabolic and catabolic reactions in the course of sepsis might add to the therapeutic toolbox of personalized care in the field of infection-driven organ dysfunction.

\section{Outlook: Resistance and tolerance responses beyond the immune system}

Evidently, resistance and tolerance responses are not restricted to the immune system. All other organs specifically respond to microbial infections. Tissue macrophages roving around the parenchyma act as sensors for invading microorganisms. Following interaction with the microbes they release signals 


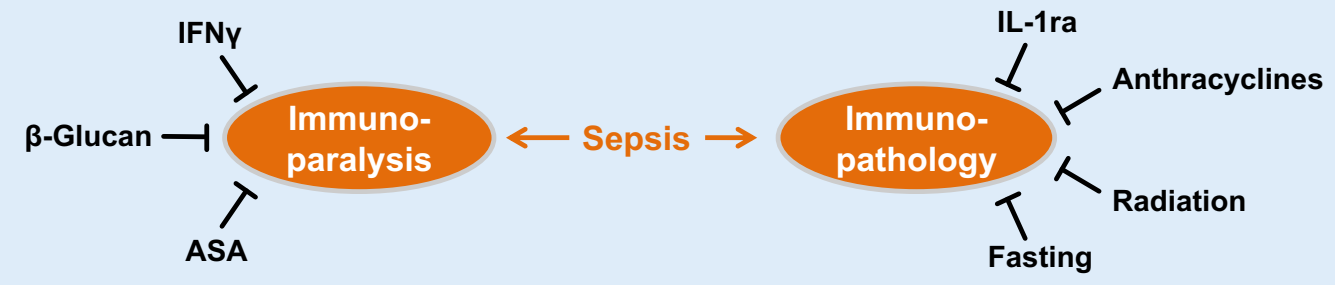

Fig. $3<$ Evolving therapeutic options to modulate resistance and tolerance responses in sepsis models. ASA acetylsalicylic acid, IFN $Y$ Interferon $\gamma$, IL-1 ra Interleukin-1 receptor antagonist which provoke defense reactions of the surrounding tissue [29]. Also non-immune cells have been shown to directly express resistance responses by attacking and destroying invading microbes. Most prominently antimicrobial peptides released by epithelial and other parenchymal cells exhibit the ability to destroy bacteria [44]. Ongoing efforts in experimental medicine are directed to use the antimicrobial capacity of peptides like defensins to treat specific infectious diseases [3]. The defense armory of parenchymal cells includes in addition phagocytosis of microbes and degradation by specific release of proteolytic enzymes or reactive oxygen species [12]. Tolerance to microbial infections is a widespread trait of parenchymal cells and organs. Originally explored in plants, tolerance responses have also been in the meantime detected in most of the parenchymal organs of mammals [2]. As a prototypical example accumulation of labile heme in plasma and urine during Plasmodium infection has been shown to induce pronounced disease tolerance to malaria. Specifically, the heme-induced tolerance response prevents the development of acute kidney injury, a clinical hallmark of severe malaria [34].

How do parenchymal organs cope with resistance and tolerance responses of the immune system? Relevant investigations reveal a tight overlap of pathogen induced metabolic and signaling reactions in immune and in parenchymal cells [12]. Unquestionably energy resources of all organs including the immune system are closely interlinked by the hormonal control of metabolites via the blood stream. Full supply of the organism with nutrients enables fervent resistance responses of the immune system but also high anabolic and proliferative capacity of parenchymal tissue. In contrast, calorie and energy deprivation might cause tolerant immune responses and catabolic maintenance reactions of the parenchyma.

In general, full regeneration of damaged tissue needs a well-balanced interplay of catabolic and anabolic events. In a first step damaged and functionless biomolecules and cellular organelles have to be degraded. Controlled proteolysis by intracellular proteasomes and extracellular proteases may take care of dysfunctional proteins [9]. Whole organelles are taken up and degraded by autophagy [36]. To fully regenerate damaged tissue these prototypical catabolic processes are mostly followed by backfilling of injured cells [39]. Here energy demanding proliferation of the surrounding tissue is necessary.

Thus, the precise adjustment of catabolic and anabolic processes is prerequisite for full regeneration of parenchymal tissue and all organs damaged either by hyperinflammation or microbial virulence in sepsis. In fact, host-pathogen interaction in sepsis comprises responses of parenchymal organs as a central, yet underestimated component. The highly dynamic tripartite interaction of pathogen, immune system and parenchyma seemingly defines the course of infection-related organ failure, i.e., sepsis and opens novel treatment options.

\section{Practical conclusion}

- Organ dysfunction results from impaired adaptation to infection which is not restricted to "hyperinflammation".

\section{- The ability to adapt to the "pathogenic load" associated} with infection is crucial for an organism and might involve "resistance" (i.e. responses of the immune system that lower pathogen burden) and "resilience" or "disease tolerance" (i.e. responses that increase the ability to cope with a persistent pathogen burden).

- Newly emerging treatment concepts that take into consideration opposing responses of immunity ("theranostics") are promising but require diagnostic tests that allow identifying various phenotypes of sepsis at the point of care.

- These strategies are currently subject to clinical trials and require more evidence for translation into clinical practice.

\section{Corresponding address}

Prof. Dr. M. Bauer
Department of Anesthesi-
ology and Intensive Care
Medicine, Jena University
Hospital
Am Klinikum 1, 07747 Jena,
Germany
Michael.Bauer@
med.uni-jena.de

Funding. Open Access funding provided by Projekt DEAL.

\section{Compliance with ethical guidelines}

Conflict of interest. M. Bauer and R. Wetzker declare that they have no competing interests.

For this article no studies with human participants or animals were performed by any of the authors. All studies performed were in accordance with the ethical standards indicated in each case. 
The supplement containing this article is not sponsored by industry.

Open Access. This article is licensed under a Creative Commons Attribution 4.0 International License, which permits use, sharing, adaptation, distribution and reproduction in any medium or format, as long as you give appropriate credit to the original author(s) and the source, provide a link to the Creative Commons licence, and indicate if changes were made. The images or other third party material in this article are included in the article's Creative Commons licence, unless indicated otherwise in a credit line to the material. If material is not included in the article's Creative Commons licence and your intended use is not permitted by statutory regulation or exceeds the permitted use, you will need to obtain permission directly from the copyright holder. To view a copy of this licence, visit http://creativecommons.org/licenses/by/4.0/.

\section{References}

1. Alers $S$ et al (2012) Role of AMPK-mTOR-Ulk1/2 in the regulation of autophagy: cross talk, shortcuts, and feedbacks. Mol Cell Biol 32:2-11

2. Ayres JS, Schneider DS (2012) Tolerance of infections. Annu Rev Immunol 30:271-294

3. Balato $G$ et al (2020) Laboratory-based versus qualitative assessment of alpha-defensin in periprosthetic hip and knee infections: a systematic review and meta-analysis. Arch Orthop Trauma Surg 140(3):293-301. https://doi.org/10. 1007/s00402-019-03232-5

4. Bauer M et al (2016) A Transcriptomic biomarker to quantify systemic inflammation in sepsis-a prospective multicenter phase ll diagnostic study. EBioMedicine6:114-125

5. Bauer $M$ et al (2018b) Deterioration of organ function as a hallmark in sepsis: the cellular perspective. Front Immunol 9:1460

6. Bauer M et al (2018a) Remembering pathogen dose: long-term adaptation in innate immunity. Trends Immunol 39:438-445

7. Beutler BA et al (1985) Cachectin/tumor necrosis factor: production, distribution, and metabolic fate in vivo. J Immunol 135:3972-3977

8. Bone RC (1996) Sir Isaac Newton, sepsis, SIRS, and CARS. Crit Care Med 24:1125-1128

9. Boukhalfa A et al (2019) Interplay between primary cilia, ubiquitin-proteasome system and autophagy. Biochimie 166:286-292. https://doi. org/10.1016/j.biochi.2019.06.009

10. Casaer MP, Van den Berghe G (2014) Nutrition in the acute phase of critical illness. N Engl J Med 370:2450-2451

11. de Castro MJ et al (2015) Nonspecific (heterologous) protection of neonatal BCG vaccination against hospitalization due to respiratory infection and sepsis. Clin Infect Dis 60:1611-1619

12. Ceponis PJ et al (2005) Epithelial cell signaling responses to enterohemorrhagic Escherichia coli infection. Mem Inst Oswaldo Cruz 100(Suppl 1):199-203

13. Cetrullo S et al (2015) mTOR, AMPK, and Sirt1: key players in metabolic stress management. Crit Rev Eukaryot Gene Expr 25:59-75

14. Chen J et al (2017) Metformin extends C. elegans lifespan through lysosomal pathway. Elife 6:e31268. https://doi.org/10.7554/eLife.31268. 001

15. Cohen J et al (2015) Sepsis: a roadmap for future research. Lancet Infect Dis 15:581-614
16. Figueiredo $\mathrm{N}$ et al (2013) Anthracyclines induce DNA damage response-mediated protection against severe sepsis. Immunity 39:874-884

17. Galic $S$ et al (2011) Hematopoietic AMPK beta1 reduces mouse adipose tissue macrophage inflammation and insulin resistance in obesity. JClin Invest 121:4903-4915

18. Grondman I et al (2019) Frontline science: Endotoxin-induced immunotolerance is associated with loss of monocyte metabolic plasticity and reduction of oxidative burst. J Leukoc Biol 106:11-25

19. Hotchkiss RS et al (2016) Sepsis and septic shock. NatRev Dis Primers 2:16045

20. Kwak HJ et al (2016) Bortezomib attenuates palmitic acid-induced ER stress, inflammation and insulin resistance in myotubes via AMPK dependent mechanism. Cell Signal 28:788-797

21. Lajqi T, Lang G-P, Haas F, Williams DL, Hudalla $H$ et al (2019) Memory-like inflammatory responses of microglia to rising doses of LPS: key role of PI3K $\gamma$. Front Immunol 10:2492

22. Leentjens J et al (2012) Reversal of immunoparalysis in humans in vivo: a double-blind, placebocontrolled, randomized pilot study. Am J Respir Crit Care Med 186:838-845

23. Leijte GP et al (2019) Treatment with acetylsalicylic acid reverses endotoxin tolerance in humans in vivo: a randomized placebo-controlled study. Crit Care Med 47:508-516

24. Li M et al (2019) Efficacy and safety of mTOR inhibitors (rapamycin and its analogues) for tuberous sclerosis complex: a meta-analysis. Orphanet JRare Dis 14:39

25. Liemburg-Apers DC et al (2016) Acute stimulation of glucose influx upon mitoenergetic dysfunction requires LKB1, AMPK, Sirt2 and mTOR-RAPTOR. JCell Sci 129:4411-4423

26. Liu X et al (2019) Rapamycin- and starvationinduced autophagy are associated with miRNA dysregulation in A549 cells. Acta Biochim Biophys Sin 51:393-401

27. Maitra U et al (2012) Molecular mechanisms responsible for the selective and low-grade induction of proinflammatory mediators in murine macrophages by lipopolysaccharide. J Immunol 189:1014-1023

28. Martin-Montalvo A et al (2013) Metformin improves healthspan and lifespan in mice. Nat Commun 4:2192

29. Mass E (2018) Delineating the origins, developmental programs and homeostatic functions of tissue-resident macrophages. Int Immunol 30:493-501

30. Miller RR 3rd et al (2018) Validation of a host response assay, SeptiCyte $L A B$, for discriminating sepsis from systemic inflammatory response syndrome in the ICU. Am J Respir Crit Care Med 198:903-913

31. Moffat C, Harper ME (2010) Metabolic functions of AMPK: aspects of structure and of natural mutations in the regulatory gamma subunits. IUBMB Life 62:739-745

32. Novakovic B et al (2016) beta-Glucan reverses the epigenetic state of LPS-induced immunological tolerance. Cell 167:1354-1368.e1314

33. Osuchowski MF et al (2012) Sepsis chronically in MARS: systemic cytokine responses are always mixed regardless of the outcome, magnitude, or phase of sepsis. JImmunol 189:4648-4656

34. Ramos $S$ et al (2019) Renal control of disease tolerance to malaria. Proc Natl Acad Sci U S A 116:5681-5686
35. Reiling JH, Sabatini DM(2006) Stress and mTORture signaling. Oncogene 25:6373-6383

36. Sanchez-Martin P, Komatsu M (2020) Physiological stress response by selective autophagy. J Mol Biol 432:53-62. https://doi.org/10.1016/j.jmb.2019. 06.013

37. Seebacher $F$ (2018) The evolution of metabolic regulation in animals. Comp Biochem Physiol B Biochem Mol Biol 224:195-203

38. Seymour CW, Angus DC (2019) Identifying sepsis phenotypes-reply. J Am Med Assoc 322:1417

39. Shukla SK et al (2019) Pharmacological control of inflammation in wound healing. J Tissue Viability 28:218-222. https://doi.org/10.1016/j.jtv.2019. 09.002

40. Singer $M$ et al (2016) The third international consensus definitions for sepsis and septic shock (Sepsis-3). JAm Med Assoc 315:801-810

41. Starr ME et al (2016) Short-term dietary restriction rescues mice from lethal abdominal sepsis and endotoxemia and reduces the inflammatory/ coagulant potential of adipose tissue. Crit Care Med 44:e509-519

42. Sutter D et al (2019) Delivery of rapamycin using in situ forming implants promotes immunoregulation and vascularized composite allograft survival. Sci Rep 9:9269

43. Tamargo-Gomez I, Marino G (2018) AMPK: regulation of metabolic dynamics in the context of autophagy. Int J Mol Sci. https://doi.org/10.3390/ ijms 19123812

44. Wah J et al (2006) Antimicrobial peptides are present in immune and host defense cells of the human respiratory and gastrointestinal tracts. Cell Tissue Res 324:449-456

45. West MA, Koons A (2008) Endotoxin tolerance in sepsis: concentration-dependent augmentation or inhibition of LPS-stimulated macrophage TNF secretion by LPS pretreatment. J Trauma 65:893-898 (discussion 898-900)

46. Zannella VE et al (2011) AMPK regulates metabolism and survival in response to ionizing radiation. Radiother Oncol 99:293-299 